\title{
White-Faced Capuchin, Cebus capucinus imitator, Hammerstone and Anvil Tool Use in Riparian Habitats on Coiba Island, Panama
}

\author{
Claudio M. Monteza-Moreno ${ }^{1,2,3,4}$ - Tamara Dogandžić ${ }^{5,6} \cdot$ Kevin A. McLean ${ }^{1,3}$. \\ Pedro L. Castillo-Caballero ${ }^{3,7}$ • Zarluis Mijango-Ramos ${ }^{3,8}$ • \\ Evelyn Del Rosario-Vargas ${ }^{3,7}$. Margaret C. Crofoot ${ }^{1,2,3,9,10,11}$. \\ Brendan J. Barrett ${ }^{3,12,13}$
}

\section{Introduction}

Tool-aided extractive foraging is an important element of the success of many dietary generalists in resource-limited ecosystems. The study of primate tool use offers insight into the possible form and function of tool use in early hominins, as well as related ecological pressures. It informs us about instances of tool use where raw materials or sites may not be preserved due to site degradation. It helps us map observable behavior onto site accumulation and increases our understanding of taphonomic biases and site formation. While habitual tool use has been reported in all great apes and many robust capuchins, it is rarer in other primates. Recently, island-living populations of long-tailed macaques (Matrivijonod et al. 2007) and gracile capuchins (Barrett et al. 2018) have been discovered to habitually use stone tools. The contrast of these tool-using insular populations relative to their mainland counterparts, and the heavy reliance on tool use in many island-living birds (Tebbich et al. 2002), suggests that some aspect of island ecology such as reduced predation, increased terrestrially, decreased dietary richness, increased ecological stress, or increased niche breadth due to reduced heterospecific competition may be conducive to the innovation, maintenance, and cultural transmission of tool use.

Although robust capuchins (Sapajus sp.) are well documented tool users, gracile capuchins (Cebus sp.), which diverged from their sister taxa ca. 6.5 mya, show little to no reliance on tool use, despite well-documented evidence of other types of socially learned traditions. The only known exception is a population of white-faced capuchins, Cebus capucinus imitator, foraging on fruits and structurally protected gastropods and crustaceans in coastal ecosystems on the Pacific island of Jicarón in Coiba National

Brendan J. Barrett

bbarrett@ab.mpg.de 
Park (CNP), Panama. Here we report a second population of stone-tool using whitefaced capuchins living on Coiba, the largest island in CNP.

\section{Site Description}

Coiba (53,582 ha) lies $30 \mathrm{~km}$ west of mainland Panama in the Pacific Ocean. This biodiversity hotspot possesses a unique floristic composition, distributed among varied habitat types. Islands in the Coiban archipelago have been isolated from the mainland since the last glacial maximum 12,000-18,000 years ago.

We first observed evidence of hammerstone and anvil tool use by capuchins on the northwest of Coiba in March 2019. During an ongoing survey of tool use across the archipelago, we observed river cobbles that had been used as hammerstones resting upon stone anvils in shallow riffles in the middle of the river and along the river bank. At nine tool sites, we observed remains of freshwater snails under hammerstones and lying on and alongside stone anvils (Fig. 1a). Tool sites were found in clusters (two or three sites separated by $<5 \mathrm{~m}$ ) as well as in isolated sites. Distances between tool sites ranged from ca. 25 to $100 \mathrm{~m}$. In April 2019, we counted 10 tool sites on a ca. 1-km transect of riverbank that lies 620-1000 m from the coast. In July 2019, we observed evidence of capuchins using hammerstones to process Bactris sp. palm fruits $c a .800 \mathrm{~m}$

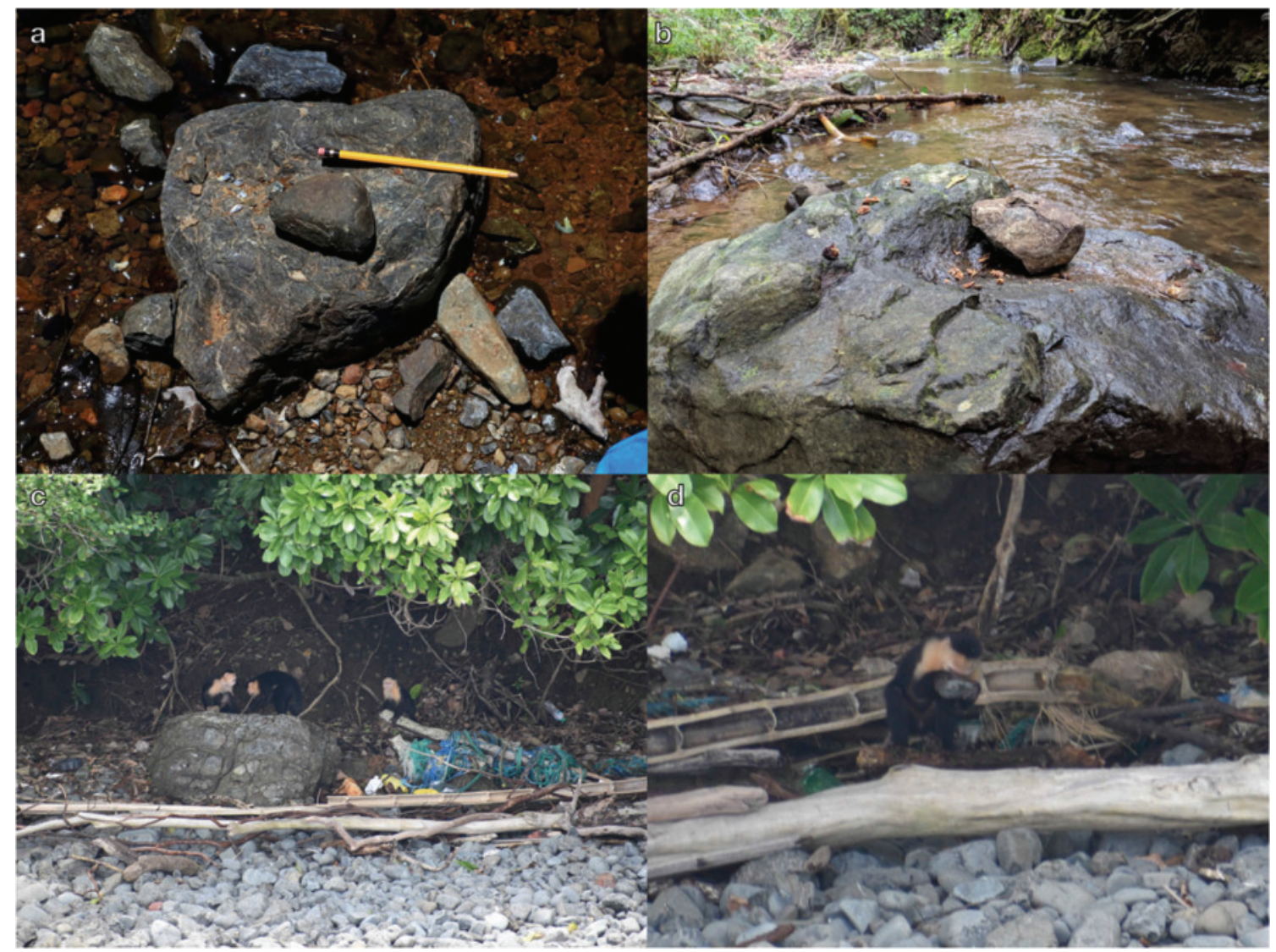

Fig. 1 (a) Hammerstone resting on anvil. Fragments of Pomacea shells lie on top of the anvil and alongside it in the river and riverbank. (b) Fragments of Bactris palm fruits under and around a hammerstone on a stone anvil in a stream bed. (c) Adult male capuchin ingesting food he processed with hammerstone on stone anvil while conspecifics observe intently. (d) Adult male capuchin using a hammerstone on a wooden anvil to process unidentified food items on the coast. Photo credits: (a) Christian Ziegler; (b) CMM; (c, d) ZMR. 
from the coast (Fig. 1b). Later we observed from a boat an adult male capuchin using hammerstones to process unidentified coastal resources accompanied by two conspecifics ca. $1300 \mathrm{~m}$ from the riparian site (Fig. 1c, d).

\section{Conclusions}

This is only the second description of hammerstone and anvil tool use in Cebus despite numerous decades-long habituated studies across their range. The only other known tool-using population is a single group $39 \mathrm{~km}$ southwest on Jicarón, the second largest island within CNP (Barrett et al. 2018). Our discovery of another population of stonetool using capuchins on a second island in the Coiba Archipelago suggests that at least two independent innovations of this behavior presumably occurred on the islands of Coiba and Jicarón since their isolation from each other and the mainland 12,00018,000 years ago. Although no dispersal events are known between the islands, this should be interpreted cautiously; Coiba and Jicarón are separated by $6.5 \mathrm{~km}$ at their closest point. This observation also supports the hypothesis that some aforementioned aspect(s) of island ecology favor the evolution and spread of tool use.

Despite significant camera trap surveys since March 2017 (see Barrett et al. 2018), we have observed stone tool use only in two geographically restricted areas of Coiba and Jicarón. The vast majority of capuchins on these islands do not appear to use stone tools, despite having access to all of the materials required to do so. Different groups across CNP in similar ecological conditions process identical resources with (Barrett et al. 2018) and without (MéndezCarvajal and Valdés-Díaz 2017) hammerstone and anvil tool use; this is consistent with stone tool use being a localized cultural tradition. Further targeted surveys on ecologically plausible sites for tool use in Coiba, and an understanding of intra- and intergroup social interactions, are necessary to explain the absence of tool use in the majority of capuchin groups in CNP.

The presence of primate stone tool use along streambeds is comparable to early hominin tool use locations in river beds or streams. In Jicaron, seasonal flooding affects site preservation in stream beds. Understanding formation of primate tool-use sites in conditions in which preservation is unlikely can provide us with testable hypotheses about the early hominin tool-use behavior and the visibility and preservation of the associated archeological record (Panger et al. 2002).

Unlike on Jicarón, we have observed tool use up to $1 \mathrm{~km}$ inland on Coiba. This new description on Coiba suggests that shallow, fast-moving streams further inland might be ideal areas to target when searching for new populations of tool-using primates. Both stone materials made available by stream flow and structurally protected invertebrates are abundant in tumultuous, riparian environments. This discovery also suggests that structurally protected resources found in streambeds and along rocky intertidal areas are equally important areas to further explore the breadth and patterning of Cebus tool use. Currently, we do not know if these coastal and riparian hammerstone and anvil tool sites on Coiba have been used by single or multiple capuchin groups. Future explorations to the site and camera trapping will be important to resolve if this tradition is confined to a single group or more widely spread. 
Acknowledgements This research was funded by an L. S. B. Leakey Foundation grant awarded to BJB as well as funds from the Max Planck Institute. It was also supported by a Packard Foundation Fellowship (201665130), a grant from the National Science Foundation (NSF BCS 1514174), and the Alexander von Humboldt Foundation in the framework of the Alexander von Humboldt Professorship endowed by the Federal Ministry of Education and Research awarded to MCC. Support was also provided by the Center for the Advanced Study of Collective Behavior at the University of Konstanz, DFG Centre of Excellence 2117 (ID: 422037984). CMM is funded by SENACYT, scholarship program number BIDP-2017-2018. This research was conducted under Ministerio de Ambiente, Panamá, scientific permit no. SC/A-23-17 and corresponding renewals and addenda. We thank the editor and two anonymous reviewers for their feedback on this manuscript.

Authors' Contributions All authors conducted fieldwork. BJB, CMM, MCC, and TD wrote the manuscript; other authors provided editorial advice.

\section{References}

Barrett, B. J., Monteza-Moreno, C. M., Dogandžić, T., Zwyns, N., Ibañez, A., \& Crofoot, M. C. (2018). Habitual stone-tool-aided extractive foraging in white-faced capuchins, Cebus capucinus. Royal Society Open Science, 5(8), 181002.

Malaivijitnond, S., Lekprayoon, C., Tandavanittj, N., Panha, S., Cheewatham, C., \& Hamada, Y. (2007). Stone-tool usage by Thai long-tailed macaques (Macaca fascicularis). American Journal of Primatology, 69(2), 227-233.

Méndez-Carvajal, P. G., \& Valdés-Díaz, S. (2017). Use of anvils and other feeding behaviors observed in Cebus imitator, Coiba Island, Panama. Tecnociencia, 19(1), 5-18.

Panger, M. A., Brooks, A. S., Richmond, B. G., \& Wood, B. (2002). Older than the Oldowan? Rethinking the emergence of hominin tool use. Evolutionary Anthropology, 11(6), 235-245.

Tebbich, S., Taborsky, M., Fessl, B., \& Dvorak, M. (2002). The ecology of tool-use in the woodpecker finch (Cactospiza pallida). Ecology Letters, 5(5), 656-664.

Ethical Note No animal care and use protocol was required for this study. The authors declare that they have no conflict of interest.

\section{Affiliations}

\section{Claudio M. Monteza-Moreno 1,2,3,4 - Tamara Dogandžić ${ }^{5,6} \cdot$ Kevin A. McLean ${ }^{1,3}$. Pedro L. Castillo-Caballero ${ }^{3,7}$ - Zarluis Mijango-Ramos ${ }^{3,8}$ • Evelyn Del Rosario-Vargas ${ }^{3,7}$ - Margaret C. Crofoot ${ }^{1,2,3,9,10,11}$ • Brendan J. Barrett ${ }^{3,12,13}$}

1 Department of Anthropology, University of California, Davis, CA, USA

2 Animal Behavior Graduate Group, University of California, Davis, CA, USA

3 Smithsonian Tropical Research Institute, Balboa, Ancón, Republic of Panama

4 Estación Científica COIBA-AIP, Ciudad del Saber, Clayton, Panamá, Republic of Panama

5 Department of Human Evolution, Max Planck Institute for Evolutionary Anthropology, Leipzig, Germany

6 Department of Anthropology, University of Pennsylvania, Philadelphia, PA, USA

7 Facultad de Ciencias Naturales, Exactas y Tecnología, Universidad de Panamá, Panamá, Republic of Panama 
8 Department of Plant Biology, University of Illinois Urbana-Champaign, Urbana, IL, USA

9 Department for the Ecology of Animal Societies, Max Planck Institute of Animal Behavior, Konstanz, Germany

10 Department of Biology, University of Konstanz, Konstanz, Germany

11 Center for the Advanced Study of Collective Behavior, University of Konstanz, Konstanz, Germany

12 Cognitive and Cultural Ecology Group, Max Planck Institute of Animal Behavior, Radolfzell, Germany

13 Department of Human Behavior, Ecology, and Culture, Max Planck Institute for Evolutionary Anthropology, Leipzig, Germany 